\title{
Sleep indices and eating behaviours in young adults: findings from Project EAT
}

\author{
Rachel P Ogilvie ${ }^{1, *}$, Pamela L Lutsey ${ }^{2}$, Rachel Widome ${ }^{2}$, Melissa N Laska ${ }^{2}$, Nicole Larson $^{2}$ \\ and Dianne Neumark-Sztainer ${ }^{2}$ \\ 'Department of Psychiatry, University of Pittsburgh School of Medicine, 3471 Fifth Avenue, Suite 1216, Kaufmann \\ Medical Building, Pittsburgh, PA 15213, USA: ${ }^{2}$ Division of Epidemiology and Community Health, University of \\ Minnesota School of Public Health; Minneapolis, MN, USA
}

Submitted 16 May 2017: Final revision received 30 0ctober 2017: Accepted 1 November 2017: First published online 6 December 2017

\begin{abstract}
Objective: To test the associations between sleep indices and eating behaviours in young adults, a group vulnerable to suboptimal sleep.

Design: Cross-sectional analysis of survey measures of sleep (i.e. time in bed, variability, timing and quality) and dietary patterns (i.e. breakfast skipping, eating at fast-food restaurants, consumption of sports and energy drinks, and sugar-free, sugar-sweetened and caffeinated beverages).

Setting: Minneapolis/St. Paul metropolitan area of Minnesota (USA).

Subjects: A total of 1854 respondents (20-30 years, $55 \cdot 6 \%$ female) from the $2008-$ 2009 survey conducted for the third wave of the population-based Project EAT (Eating and Activity in Teens and Young Adults) study.

Results: After adjustment for demographic and behavioural covariates in linear regression models, those who went to bed after 00.30 hours consumed 0.3 more servings of sugar-sweetened beverages per day, consumed 1.7 times more energy drinks, skipped breakfast 1.8 more times per week and consumed fast food 0.3 more times per week compared with those who went to bed before 22.30 hours. Reported sleep quality in the lowest (Q1) $v$. highest (Q3) tertile was associated with more intake of energy drinks (Q3 $v$. Q1, prevalence ratio, 95\% CI: 1·79, 1.24, $2 \cdot 34)$, sports drinks $(1.28,1 \cdot 00,1.55)$ and breakfast skipping (adjusted mean, $95 \%$ CI: Q1: 4.03, 3.81, 4.26; Q3: 3.43, 3.17, 3.69). Time in bed and sleep variability were associated with few eating behaviours.

Conclusions: Some, but not all, sleep indices were related to problematic eating behaviours. Sleep habits may be important to address in interventions and policies that target improvements in eating patterns and health outcomes.
\end{abstract}

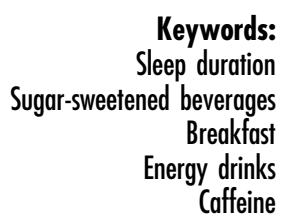

Several organizations currently recommend that adults sleep for at least $7 \mathrm{~h}$ per night, including the American Academy of Sleep Medicine and Sleep Research Society, the National Sleep Foundation and the Centers for Disease Control and Prevention ${ }^{(1-3)}$. Failing to meet this recommendation has been associated with poor physical health $^{(4)}$, mental health ${ }^{(5)}$ and quality of life ${ }^{(6-8)}$. National survey data indicate that young adults aged 25-34 years are particularly likely to get insufficient sleep; in 2014, $28 \%$ reported sleeping fewer than $7 \mathrm{~h}$ per night ${ }^{(9)}$. Although short sleep duration and its relationship to dietary intake has been examined in adolescents ${ }^{(10,11)}$, there is scant research on this relationship in young adults, who are undergoing major life transitions and engaging in independent decision making for the first time.

Most research among adults has involved short-term experiments conducted in sleep labs ${ }^{(12)}$, which do not provide information on habitual behaviours among freeliving adults. The few observational studies of adults have examined how sleep duration may be related to obesity $^{(13,14)}$ and energy intake, with inconsistent results, but the specific aspects and patterns of dietary intake correlated with short sleep duration have scarcely been explored $^{(15-17)}$. Even less is known about the association of sleep quality, variability and timing with dietary factors ${ }^{(18)}$. Furthermore, most prior studies were conducted in predominantly white populations, despite previous research exposing racial/ethnic ${ }^{(19-21)}$ and socio-economic differences in sleep duration ${ }^{(22,23)}$. A recent American Heart Association scientific statement named the inclusion of more diverse populations a top sleep research priority ${ }^{(24)}$.

The Project EAT (Eating and Activity in Teens and Young Adults) study provides an opportunity to investigate the potentially bidirectional relationship between 
several sleep indices and dietary factors in a racially, ethnically and socio-economically diverse population of young adults. A previous analysis of Project EAT data, which used data from the same 'wave' presented herein, found that short sleep duration was associated with higher BMI in men but not women ${ }^{(25)}$. We hypothesized that young adults who reported inadequate $v$. recommended amounts of sleep would consume more caffeinated and sugar-sweetened beverages and report more frequent breakfast skipping and eating at fast-food restaurants.

\section{Methods}

Project EAT was designed to study dietary intake, physical activity and weight among young people. Baseline data were collected in 1998-1999, when 4746 middle- and high-school students aged 11-18 years from thirty-one socio-economically and racially/ethnically diverse schools in the Minneapolis/St. Paul metropolitan area of Minnesota (USA) completed questionnaires and anthropometric measures $^{(26)}$. A 10-year follow-up survey (EAT-III) was completed in 2008-2009 by mailing all original participants an invitation to complete questionnaires on paper or online. A total of 2287 young adults completed this third wave of data collection, representing $66.4 \%$ of those who could be contacted $^{(27)}$. At the time of EAT-III, participants were 20-30 years old. For the present analysis, we limited the sample to participants whose third-wave survey data included plausible reports of dietary intake and sleep ( $n$ 1854). The University of Minnesota Institutional Review Board approved all study protocols and participants provided informed consent.

The original Project EAT survey was modified for EATIII to improve the relevance of items for young adults and to investigate new research areas. Focus groups tested an initial draft and feedback was used to alter problematic survey measures and to expand on areas of importance. A revised survey was tested in a different sample to examine test-retest reliability over 1 to 3 weeks. Additional details of the survey development process have been described elsewhere ${ }^{(28)}$.

\section{Sleep variables}

Participants were asked about their usual bedtime and wake time on both weekdays and weekends, which were used to calculate average weekday and weekend 'time in bed $^{\text {,25) }}$. These items were drawn from a questionnaire previously used in studies of adolescent sleep ${ }^{(29,30)}$, and similar questions have been significantly correlated with both sleep diaries and actigraphy ${ }^{(31)}$. Average daily time in bed was calculated using the following formula: (weekday time in bed $\times 5 / 7)+($ weekend time in bed $\times 2 / 7)$. The calculation of hours of sleep per day assumed that wake time occurred after bed time. To correct problems with $\mathrm{am} / \mathrm{pm}$, sleep times longer than $16 \mathrm{~h}$ were adjusted by subtracting by 12 to obtain the value if the correct am/pm designation had been selected. Times in bed less than $4 \mathrm{~h}$ were set to missing. Time in bed was modelled categorically ( $<7 \mathrm{~h}, 7-<8 \mathrm{~h}, 8-<9 \mathrm{~h}$ and $\geq 9 \mathrm{~h}$ per night).

We also examined 'sleep variability' by calculating the absolute value of the difference between weekday and weekend time in bed, which was modelled in quartiles $(<0.5 \mathrm{~h}, 0.5-<1 \mathrm{~h}, 1-1.5 \mathrm{~h},>1.5 \mathrm{~h})$. 'Sleep timing' was measured by averaging weekend and weekday bedtimes and modelling them in four categories (before 22.30 hours, 22.30-23.30 hours, 23.30-00.30 hours and after 00.30 hours). 'Sleep quality' was measured using the following question on the Kandel and Davies depressive symptoms questionnaire ${ }^{(32)}$ : 'During the past 12 months, how often have been bothered or troubled by having trouble going to sleep or staying asleep?' (test-retest $r=0 \cdot 64)$. Possible responses to this question included 'not at all', 'sometimes' and 'very much'.

\section{Dietary variables}

Questions on frequency of skipping breakfast and eating at a fast-food restaurant were assessed on the Project EATIII survey. Breakfast was assessed with the following question: 'During the past week, how many days did you eat breakfast?' with five possible responses ranging from 'never' to 'every day' (test-retest $r=0 \cdot 82$ ). Fast food was assessed with the following question: 'In the past week, how often did you eat something from a fast food restaurant (like McDonald's, Burger King, Hardee's etc.)?' (test-retest $r=0 \cdot 48$ ). Six possible responses were given, ranging from 'never' to 'more than 7 times'. Both variables were treated as continuous.

Questions on energy and sports drink consumption were also assessed on the Project EAT-III survey. Energy drink consumption was assessed with the following question: 'In the past year, how many times did you usually drink an energy drink (such as Red Bull, Full Throttle, Rockstar, etc.)?' Sports drink consumption was assessed with the following question: 'In the past year how many times did you usually drink a sports drink (such as Gatorade, PowerAde, etc.)?' Seven possible responses were given, ranging from 'less than once per month' to ' 2 or more per day'. Based on the distribution of the variables $^{(33)}$, energy and sports drinks were dichotomized into two categories: at least one drink per week and less than one drink per week (test-retest agreement $=94 \%$ for sports drinks, $97 \%$ for energy drinks).

Information on beverages including sugar-sweetened beverages, sugar-free beverages and caffeinated beverages was taken from a semi-quantitative FFQ that was administered at the same time as the Project EAT-III survey. This FFQ measured multivitamins, dietary supplements and intakes of 151 foods. The reproducibility and validity compared with diet records for measuring beverages have been assessed, and moderate to high correlations have been found (mean $r$ for reproducibility $=0.59$, 
mean $r$ for validity $=0.63)^{(34)}$. Sugar-sweetened, sugar-free and caffeinated beverages were assessed with nine response categories, ranging from 'never or less than once a month' to ' $6+$ per day'. This was translated into daily servings with a single serving defined as one glass, bottle or can. The sugar-sweetened beverages variable was created by summing the responses to questions on carbonated beverages with caffeine and sugar (e.g. Coke, Pepsi, Mountain Dew, Dr. Pepper), other carbonated beverages with sugar (e.g. 7-Up, Root Beer, Ginger Ale, Caffeine-Free Coke) and other sugared beverages (punch, lemonade, sports drinks or sugared ice tea). The sugar-free beverages variable was created by summing responses to questions on low-calorie beverages with caffeine (e.g. Diet Coke, Diet Mountain Dew) and other low-calorie beverages without caffeine (e.g. Diet 7-Up). The caffeinated beverages variable was created by summing the responses to the questions on low-calorie beverages with caffeine and carbonated beverages with caffeine and sugar, along with additional items that assessed intake of tea with caffeine including green tea ( 8 fluid ounces $(237 \mathrm{ml})$ ), coffee with caffeine (8 fluid ounces $(237 \mathrm{ml})$ ) and dairy coffee drink (e.g. cappuccino, 16 fluid ounces $(473 \mathrm{ml})$ ). These variables were treated as continuous.

\section{Covariates}

Demographic characteristics including sex, ethnicity/race, age, education and marital status were self-reported. Depressive symptoms were assessed via a six-item scale validated for use in young people ${ }^{(32)}$. These six items asked about tiredness, difficulty sleeping, sadness, hopelessness, nervousness and worry over the past year, and were scored from 1 to 3 . For the present analysis, we excluded two items related to sleep in the calculation of depressive symptoms and summed and categorized into approximate quartiles the remaining items on sadness, hopelessness, nervousness and worry. Physical activity was assessed with via three items from the Godin Leisuretime Exercise questionnaire, which asked about hours of mild, moderate and strenuous exercise in a usual week ${ }^{(35)}$. Responses to these questions were used to calculate moderate-vigorous leisure activity in hours, which was modelled continuously. BMI was calculated via selfreported height and weight, which was strongly correlated with anthropometric measurements in a validation study during an earlier wave of Project EAT ${ }^{(36)}$. Alcohol was measured in grams. These variables were included as covariates due to their bidirectional relationships with sleep $^{(37,38)}$.

\section{Analysis}

Descriptive statistics were calculated to examine sociodemographic and behavioural characteristics by category of time in bed. Based on the outcome, either linear or logistic regression was used to model the cross-sectional relationship between each sleep exposure and each dietary outcome. Adjusted probabilities standardized to the total population were calculated for each sleep category in the logistic models, and these probabilities were used to calculate prevalence ratios for each outcome. Model 1 adjusted for age, sex, race/ethnicity, education and marital status, while Model 2 added depressive symptoms and physical activity. For models with sleep variability, timing and quality as the exposure, Model 3 added time in bed. Effect modification was also examined by gender, as previous research has found different relationships between sleep habits and dietary intake for men and women ${ }^{(39)}$. Two sensitivity analyses were performed: one where we utilized an additional time in bed category of $<6 \mathrm{~h}$ per night and one where we adjusted for BMI. All analyses used inverse probability weighting to account for differential loss to follow-up ${ }^{(40)}$.

\section{Results}

The mean age in the analytic sample was 25.4 (SD 1.7) years and $55.6 \%$ were female. Participants self-reported sleeping a mean of 8.3 (SD 1.2) h per night. The distribution of sleep times was: $11.5 \%$ slept $<7$ h per night, $26.6 \%$ slept $7-<8 \mathrm{~h}$, $36 \cdot 2 \%$ slept $8-<9 \mathrm{~h}$ and $25 \cdot 8 \%$ slept $\geq 9 \mathrm{~h}$ per night. Regarding daily dietary intake, on average, participants consumed 0.9 servings of sugar-sweetened beverages, 0.7 servings of caffeinated beverages and 0.4 servings of sugarfree beverages. Per week, participants consumed breakfast an average of 3.9 times and ate something from a fast-food restaurant on 1.6 occasions. The proportion of the sample that reported consuming at least one energy drink per week was $18.0 \%$, and consuming at least one sports drink per week was reported by $30 \cdot 2 \%$.

Table 1 shows sociodemographic and behavioural characteristics by time in bed category. Those who slept $<7 \mathrm{~h}$ per night were more likely to be male, non-white, have less formal education and have a higher mean score for depressive symptoms relative to those who slept longer. Those with later bedtimes were more likely to be male, have less formal education and report higher mean depressive symptoms. Good sleep quality was more common among men and those with more education (data not shown).

Table 2 shows the mean intakes of beverages and mean frequency of skipping breakfast and eating at a fast-food restaurant by categories of sleep variables (time in bed, variability, timing and quality). After adjustment for demographics, some associations were found between sleep indices and eating behaviours, particularly for sleep timing, although many associations were not significant. Those who went to bed after 00.30 hours consumed $0 \cdot 3$ more servings of sugar-sweetened beverages per day, skipped breakfast 1.8 more times per week and consumed fast food 0.3 more times per week compared with those who went to bed before 22.30 hours. No strong associations were found between sleep timing and caffeinated or 
Table 1 Participant characteristics by sleep duration category: young adults aged 20-30 years, Minneapolis/St. Paul metropolitan area of Minnesota, USA, 2008-2009, Project EAT (Eating and Activity in Teens and Young Adults)

\begin{tabular}{|c|c|c|c|c|c|c|c|c|}
\hline & \multicolumn{2}{|c|}{$\begin{array}{c}<7 \text { h per night } \\
(N 203 ; 11.5 \%)\end{array}$} & \multicolumn{2}{|c|}{$\begin{array}{c}7-<8 \mathrm{~h} \text { per night } \\
(N 471 ; 26.6 \%)\end{array}$} & \multicolumn{2}{|c|}{$\begin{array}{c}8-<9 \text { h per night } \\
(N 642 ; 36.2 \%)\end{array}$} & \multicolumn{2}{|c|}{$\begin{array}{l}\geq 9 \text { h per night } \\
(N 457 ; 25.8 \%)\end{array}$} \\
\hline & Mean or $n$ & SD or $\%$ & Mean or $n$ & SD or $\%$ & Mean or $n$ & SD or $\%$ & Mean or $n$ & SD or $\%$ \\
\hline \multicolumn{9}{|l|}{ Demographics } \\
\hline Age (years), mean and SD & 25.5 & $1 \cdot 6$ & $25 \cdot 6$ & $1 \cdot 6$ & $25 \cdot 3$ & $1 \cdot 6$ & $25 \cdot 1$ & $1 \cdot 7$ \\
\hline \multicolumn{9}{|l|}{ Race/ethnicity $†, n$ and $\%$} \\
\hline White & 93 & $9 \cdot 8$ & 255 & $27 \cdot 0$ & 384 & $40 \cdot 6$ & 214 & $22 \cdot 6$ \\
\hline Asian & 39 & $12 \cdot 3$ & 90 & 28.5 & 92 & $29 \cdot 1$ & 95 & $30 \cdot 1$ \\
\hline Black & 44 & $16 \cdot 1$ & 64 & $23 \cdot 1$ & 82 & $29 \cdot 6$ & 86 & $31 \cdot 1$ \\
\hline Hispanic & 9 & $10 \cdot 0$ & 27 & $30 \cdot 2$ & 37 & $40 \cdot 8$ & 17 & $19 \cdot 1$ \\
\hline Mixed/Other & 15 & $12 \cdot 1$ & 31 & 23.9 & 43 & $33 \cdot 4$ & 39 & $30 \cdot 6$ \\
\hline \multicolumn{9}{|l|}{ Education $\dagger, n$ and $\%$} \\
\hline Less than high school & 11 & $16 \cdot 2$ & 8 & $11 \cdot 2$ & 18 & $25 \cdot 7$ & 32 & $46 \cdot 9$ \\
\hline High school/GED & 86 & $12 \cdot 8$ & 178 & $26 \cdot 6$ & 216 & $32 \cdot 2$ & 191 & 28.4 \\
\hline Vocational or associates degree & 60 & $13 \cdot 4$ & 115 & $25 \cdot 6$ & 153 & 33.9 & 122 & $27 \cdot 1$ \\
\hline College graduate & 45 & $7 \cdot 8$ & 169 & $29 \cdot 0$ & 255 & $43 \cdot 9$ & 112 & $19 \cdot 2$ \\
\hline Married, $n$ and $\%$ & 38 & $18 \cdot 6$ & 131 & $28 \cdot 1$ & 166 & 25.9 & 83 & $18 \cdot 1$ \\
\hline \multicolumn{9}{|l|}{ Behavioural characteristics, mean and SD } \\
\hline Physical activity (h MVPA/week) & 4.4 & $4 \cdot 1$ & $4 \cdot 1$ & 3.9 & $4 \cdot 3$ & $4 \cdot 0$ & 3.5 & 3.6 \\
\hline Depression scale & $19 \cdot 2$ & $4 \cdot 7$ & $17 \cdot 9$ & $5 \cdot 1$ & $18 \cdot 0$ & 4.9 & $19 \cdot 0$ & $4 \cdot 6$ \\
\hline BMI $\left(\mathrm{kg} / \mathrm{m}^{2}\right)$, mean and SD & $27 \cdot 6$ & $5 \cdot 5$ & $26 \cdot 3$ & $5 \cdot 6$ & $26 \cdot 1$ & $6 \cdot 3$ & $26 \cdot 6$ & $6 \cdot 1$ \\
\hline \multicolumn{9}{|l|}{ Outcomes } \\
\hline Caffeinated drinks per day $\ddagger$ & 0.9 & $1 \cdot 2$ & 0.7 & $1 \cdot 0$ & 0.7 & $1 \cdot 0$ & 0.7 & $1 \cdot 1$ \\
\hline Sugar-sweetened beverages per day§ & $1 \cdot 1$ & $1 \cdot 3$ & 0.8 & $1 \cdot 2$ & 0.8 & $1 \cdot 1$ & 0.9 & $1 \cdot 4$ \\
\hline Sugar-free beverages per day $\|$ & 0.4 & $1 \cdot 0$ & 0.4 & 0.8 & 0.4 & 0.8 & 0.3 & 0.8 \\
\hline Breakfast consumption per week & 3.6 & $2 \cdot 2$ & 3.9 & 2.4 & $4 \cdot 2$ & $2 \cdot 5$ & 3.6 & 2.4 \\
\hline Fast food consumption per week & $1 \cdot 7$ & 1.7 & 1.5 & 1.7 & 1.5 & $1 \cdot 7$ & $1 \cdot 7$ & 1.6 \\
\hline Energy drinks $\mathbb{I}$ & 52 & $25 \cdot 7$ & 79 & $16 \cdot 9$ & 111 & $17 \cdot 3$ & 76 & $16 \cdot 6$ \\
\hline Sports drinksף & 76 & $37 \cdot 4$ & 161 & 34.2 & 178 & 27.7 & 120 & 26.4 \\
\hline
\end{tabular}

GED, General Educational Development; MVPA, moderate-vigorous physical activity.

†Row percentages.

¥Caffeinated beverages were defined as low-calorie beverages with caffeine (e.g. Diet Coke, Diet Mountain Dew), carbonated beverages with caffeine and sugar (e.g. Coke, Pepsi, Mountain Dew, Dr. Pepper), tea with caffeine including green tea, coffee with caffeine and dairy coffee drink (e.g. cappuccino).

§Sugar-sweetened beverages were defined as carbonated beverages with caffeine and sugar, other carbonated beverages with sugar (e.g. 7-Up, Root Beer, Ginger Ale, Caffeine-Free Coke) and other sugared beverages (punch, lemonade, sports drinks or sugared ice tea).

|| Sugar-free beverages were defined as low-calorie beverages with caffeine and other low-calorie beverages without caffeine (e.g. Diet 7-Up).

IEnergy and sports drink consumption defined as at least one drink per week $v$. less than one drink per week.

sugar-free beverages. Results were similar after adjustment for depressive symptoms, physical activity, alcohol and time in bed.

For the remaining sleep indices, there were few strong statistically significant associations with the continuous eating behaviours after adjustment for demographics. Compared with those who slept $7-<8 \mathrm{~h}$ per night, those who slept $<7 \mathrm{~h}$ consumed an average of approximately $0 \cdot 2$ more servings of caffeinated beverages per day ( 0.87 ( $95 \%$ CI $0.71,1.04)$ servings $v .0 .66$ (95\% CI 0.57, 0.75) servings; $P=0.03)$. Compared with those with low sleep variability, those in the highest sleep variability quartile consumed fast food 0.3 more times per week $(P=0 \cdot 02)$. As an indicator of sleep quality, those reporting 'very much' difficulty falling/ staying asleep skipped breakfast approximately one additional time every $10 \mathrm{~d}$ compared to those who reported no trouble falling/staying asleep $(P<0 \cdot 001)$.

Effect modification by sex was also examined and stratified analyses for significant multiplicative interaction terms can be found in Table 3. Although the frequency with which men skipped breakfast was unrelated to time in bed, women who slept $<7 \mathrm{~h}$ per night skipped breakfast nearly one additional time per week compared with women who slept $7-<8 \mathrm{~h}$ ( $P$ for interaction $=0 \cdot 01$ ). Men who went to bed after 00.30 hours consumed 0.5 more caffeinated beverages and 0.25 more sugar-free beverages daily than men who went to bed before 22.30 hours, while women consumed approximately the same amount of those beverages regardless of bedtime ( $P$ for interaction $<0 \cdot 01$ ).

Table 4 shows prevalence ratios (PR) for intakes of energy and sports drinks (modelled dichotomously: at least one drink per week and less than one drink per week) by sleep exposure category. After adjustment for demographics, those who slept fewer hours, had more sleep variability, reported later bedtimes and reported 'very much' difficulty falling/ staying asleep were more likely to consume energy drinks, although the associations were not statistically significant across all models. For sleep timing, those who reported going to bed after 00.30 hours were 1.83 (95\% CI 1.10, 2.55) times more likely to consume at least one energy drink per week than those who went to bed at 22.30 hours or earlier. Those who reported $\geq 8 \mathrm{~h}$ of sleep were less likely to consume sports drinks than those who slept $7-<8$ h per night, while those who reported 'very much' difficulty falling/staying 
Table 2 Adjusted mean dietary intakes $(95 \% \mathrm{Cl}$ ) by categories of sleep duration, variability, timing and quality: young adults aged $20-30$ years, Minneapolis/St. Paul metropolitan area of Minnesota, USA, 2008-2009, Project EAT (Eating and Activity in Teens and Young Adults)

\begin{tabular}{|c|c|c|c|c|c|c|c|c|}
\hline & \multicolumn{8}{|c|}{ Time in bed } \\
\hline & \multicolumn{2}{|c|}{$\begin{array}{l}<7 \text { h per night } \\
(N 203)\end{array}$} & \multicolumn{2}{|c|}{$\begin{array}{l}7-<8 \mathrm{~h} \text { per night } \\
(N 471) \text { (referent) }\end{array}$} & \multicolumn{2}{|c|}{$\begin{array}{c}8-<9 \mathrm{~h} \text { per night } \\
(N \mathrm{642})\end{array}$} & \multicolumn{2}{|c|}{$\begin{array}{l}\geq 9 \text { h per night } \\
\quad(N \text { 457) }\end{array}$} \\
\hline & Mean & $95 \% \mathrm{Cl}$ & Mean & $95 \% \mathrm{Cl}$ & Mean & $95 \% \mathrm{Cl}$ & Mean & $95 \% \mathrm{Cl}$ \\
\hline \multicolumn{9}{|c|}{ Caffeinated drinks $†$} \\
\hline Model 1 & $0.87^{\star}$ & $0.71,1.04$ & 0.66 & $0.57,0.75$ & 0.68 & $0.60,0.77$ & 0.74 & $0.63,0.85$ \\
\hline Model 2 & $0.87^{\star}$ & $0.71,1.04$ & 0.67 & $0.58,0.76$ & 0.70 & $0.61,0.78$ & 0.71 & $0.61,0.82$ \\
\hline \multicolumn{9}{|c|}{ Sugar-sweetened beveragesł } \\
\hline Model 1 & 0.99 & $0.79,1.18$ & 0.85 & $0.74,0.97$ & 0.83 & $0.73,0.93$ & 0.94 & $0.79,1.09$ \\
\hline Model 2 & 0.99 & $0.80,1 \cdot 17$ & 0.86 & $0.74,0.98$ & 0.85 & $0.75,0.94$ & 0.91 & $0.76,1.05$ \\
\hline \multicolumn{9}{|c|}{ Sugar-free beverages§ } \\
\hline Model 1 & 0.47 & $0.33,0.62$ & 0.36 & $0.29,0.43$ & 0.33 & $0.28,0.39$ & 0.33 & $0.26,0.40$ \\
\hline Model 2 & 0.47 & $0.32,0.61$ & 0.36 & $0.29,0.43$ & 0.34 & $0.28,0.40$ & 0.32 & $0.25,0.39$ \\
\hline \multicolumn{9}{|c|}{ Breakfast consumption } \\
\hline Model 1 & 3.78 & $3 \cdot 41,4 \cdot 14$ & 3.94 & $3.72,4.15$ & $4 \cdot 07$ & $3.87,4.27$ & 3.66 & $3.39,3.92$ \\
\hline Model 2 & 3.77 & $3 \cdot 42,4 \cdot 12$ & 3.92 & $3.70,4 \cdot 14$ & 4.04 & $3.84,4.24$ & 3.69 & $3.43,3.95$ \\
\hline \multicolumn{9}{|c|}{ Fast-food consumption } \\
\hline Model 1 & 1.56 & $1 \cdot 26,1 \cdot 85$ & 1.53 & $1.38,1.69$ & 1.58 & $1.45,1.71$ & 1.72 & $1.56,1.89$ \\
\hline \multirow[t]{4}{*}{ Model 2} & 1.56 & $1 \cdot 27,1 \cdot 85$ & 1.55 & $1.39,1.70$ & 1.60 & $1.47,1.73$ & 1.71 & $1.54,1.88$ \\
\hline & \multicolumn{8}{|c|}{ Sleep variability } \\
\hline & \multicolumn{2}{|c|}{$\begin{array}{c}<0.5 \mathrm{~h} \\
(N 339) \text { (referent) }\end{array}$} & \multicolumn{2}{|c|}{$\begin{array}{l}0.5-<1 \mathrm{~h} \\
(N 364)\end{array}$} & \multicolumn{2}{|c|}{$\begin{array}{l}1-1.5 \mathrm{~h} \\
(N 571)\end{array}$} & \multicolumn{2}{|c|}{$\begin{array}{l}>1.5 \mathrm{~h} \\
(N 200)\end{array}$} \\
\hline & Mean & $95 \% \mathrm{Cl}$ & Mean & $95 \% \mathrm{Cl}$ & Mean & $95 \% \mathrm{Cl}$ & Mean & $95 \% \mathrm{Cl}$ \\
\hline \multicolumn{9}{|c|}{ Caffeinated drinks } \\
\hline Model 1 & 0.71 & $0.59,0.84$ & 0.72 & $0.60,0.83$ & 0.67 & $0.59,0.75$ & 0.76 & $0.66,0.86$ \\
\hline Model 2 & 0.72 & $0.60,0.84$ & 0.71 & $0.60,0.83$ & 0.68 & $0.60,0.77$ & 0.75 & $0.66,0.84$ \\
\hline Model 3 & 0.72 & $0.59,0.84$ & 0.71 & $0.60,0.83$ & 0.69 & $0.60,0.77$ & 0.75 & $0.65,0.84$ \\
\hline Sugar-swee & & & & & & & & \\
\hline Model 1 & 0.95 & $0.79,1.10$ & 0.86 & $0.73,1.00$ & 0.86 & $0.74,0.98$ & 0.88 & $0.76,0.99$ \\
\hline Model 2 & 0.95 & $0.80,1 \cdot 10$ & 0.86 & $0.73,0.99$ & 0.88 & $0.76,1.00$ & $0 \cdot 86$ & $0.75,0.97$ \\
\hline Model 3 & 0.95 & $0.79,1.10$ & 0.86 & $0.73,0.99$ & 0.88 & $0.76,1.00$ & 0.86 & $0.75,0.97$ \\
\hline Sugar-free & & & & & & & & \\
\hline Model 1 & 0.29 & $0.22,0.36$ & 0.38 & $0.29,0.47$ & 0.34 & $0.28,0.40$ & $0.39^{*}$ & $0.32,0.47$ \\
\hline Model 2 & 0.30 & $0.22,0.37$ & 0.38 & $0.29,0.47$ & 0.35 & $0.28,0.41$ & 0.39 & $0.31,0.46$ \\
\hline Model 3 & 0.30 & $0.23,0.38$ & 0.38 & $0.29,0.47$ & 0.35 & $0.28,0.41$ & 0.39 & $0.31,0.46$ \\
\hline Breakfast c & & & & & & & & \\
\hline Model 1 & 3.81 & $3 \cdot 52,4 \cdot 10$ & 4.09 & $3.85,4.33$ & 3.95 & $3 \cdot 74,4 \cdot 17$ & 3.74 & $3.51,3.97$ \\
\hline Model 2 & 3.77 & $3.49,4.06$ & 4.09 & $3.85,4.33$ & 3.93 & $3.71,4.15$ & 3.77 & $3.54,4.00$ \\
\hline Model 3 & 3.81 & $3 \cdot 52,4 \cdot 10$ & 4.07 & $3.83,4.31$ & 3.94 & $3.72,4.16$ & 3.75 & $3.52,3.98$ \\
\hline Fast food ce & & & & & & & & \\
\hline Model 1 & 1.47 & $1.30,1.64$ & 1.60 & $1.41,1.79$ & 1.53 & $1.40,1.67$ & $1.77^{\star}$ & $1.60,1.93$ \\
\hline Model 2 & 1.49 & $1.31,1.66$ & 1.61 & $1.42,1.79$ & 1.55 & $1.41,1.68$ & $1.76^{\star}$ & $1.59,1.93$ \\
\hline Model 3 & 1.45 & $1.27,1.63$ & 1.62 & $1.43,1.80$ & 1.55 & $1.41,1.68$ & $1.77^{\star}$ & $1.60,1.94$ \\
\hline & & & & Slee & timing & & & \\
\hline & $\begin{array}{r}\leq 2 \\
(N 32\end{array}$ & $\begin{array}{l}0 \text { hours } \\
\text { (referent) }\end{array}$ & 22.30 & $\begin{array}{l}30 \text { hours } \\
197)\end{array}$ & 23.30 & $\begin{array}{l}30 \text { hours } \\
390)\end{array}$ & & $\begin{array}{l}\text { hours } \\
60 \text { ) }\end{array}$ \\
\hline & Mean & $95 \% \mathrm{Cl}$ & Mean & $95 \% \mathrm{Cl}$ & Mean & $95 \% \mathrm{Cl}$ & Mean & $95 \% \mathrm{Cl}$ \\
\hline Caffeinated & & & & & & & & \\
\hline Model 1 & 0.64 & $0.53,0.75$ & 0.66 & $0.57,0.75$ & 0.72 & $0.61,0.82$ & $0.80^{*}$ & $0.71,0.90$ \\
\hline Model 2 & 0.66 & $0.55,0.77$ & 0.67 & $0.57,0.76$ & 0.71 & $0.61,0.82$ & 0.79 & $0.69,0.88$ \\
\hline Model 3 & 0.67 & $0.56,0.78$ & 0.68 & $0.58,0.77$ & 0.71 & $0.61,0.82$ & 0.77 & $0.68,0.87$ \\
\hline Sugar-swee & & & & & & & & \\
\hline Model 1 & 0.73 & $0.60,0.87$ & 0.78 & $0.68,0.87$ & 0.88 & $0.75,1.02$ & $1.05^{\star *}$ & $0.93,1.18$ \\
\hline Model 2 & 0.75 & $0.61,0.88$ & 0.79 & $0.69,0.90$ & 0.88 & $0.75,1.01$ & $1.04^{\star *}$ & $0.92,1.16$ \\
\hline Model 3 & 0.75 & $0.61,0.88$ & 0.80 & $0.69,0.90$ & 0.88 & $0.75,1.02$ & $1.04^{\star \star}$ & $0.91,1.16$ \\
\hline Sugar-free & & & & & & & & \\
\hline Model 1 & 0.36 & $0.28,0.45$ & 0.32 & $0.26,0.39$ & 0.38 & $0.29,0.47$ & 0.36 & $0.29,0.43$ \\
\hline Model 2 & 0.38 & $0.29,0.46$ & 0.33 & $0.27,0.39$ & 0.38 & $0.29,0.47$ & 0.35 & $0.29,0.42$ \\
\hline Model 3 & 0.39 & $0.31,0.48$ & 0.34 & $0.27,0.40$ & 0.37 & $0.28,0.46$ & 0.34 & $0.27,0.41$ \\
\hline
\end{tabular}




\begin{tabular}{|c|c|c|c|c|c|c|c|c|}
\hline & \multicolumn{8}{|c|}{ Sleep timing } \\
\hline & \multicolumn{2}{|c|}{$\begin{array}{c}\leq 22.30 \text { hours } \\
(N 327) \text { (referent) }\end{array}$} & \multicolumn{2}{|c|}{$\begin{array}{c}22.30-23.30 \text { hours } \\
(N 497)\end{array}$} & \multicolumn{2}{|c|}{$\begin{array}{c}23.30-00.30 \text { hours } \\
(N 390)\end{array}$} & \multicolumn{2}{|c|}{$\begin{array}{l}>00.30 \text { hours } \\
(N 560)\end{array}$} \\
\hline & Mean & $95 \% \mathrm{Cl}$ & Mean & $95 \% \mathrm{Cl}$ & Mean & $95 \% \mathrm{Cl}$ & Mean & $95 \% \mathrm{Cl}$ \\
\hline \multicolumn{9}{|c|}{ Breakfast consumption } \\
\hline Model 1 & 4.98 & $4 \cdot 70,5 \cdot 25$ & $4 \cdot 11^{\star \star \star}$ & $3.88,4.35$ & $3 \cdot 73^{\star \star \star}$ & $3.48,3.98$ & $3 \cdot 18^{\star \star \star}$ & $2.97,3.39$ \\
\hline Model 2 & 4.94 & $4 \cdot 66,5 \cdot 22$ & $4 \cdot 10^{\star \star \star}$ & $3.87,4.33$ & $3 \cdot 73^{\star \star \star}$ & $3.48,3.98$ & $3 \cdot 21^{\star \star \star}$ & $3.00,3.42$ \\
\hline Model 3 & 4.99 & $4 \cdot 70,5 \cdot 27$ & $4 \cdot 11^{\star \star \star}$ & $3.87,4.34$ & $3 \cdot 71^{\star \star \star}$ & $3.47,3.96$ & $3 \cdot 19^{\star \star \star}$ & $2.97,3.40$ \\
\hline \multicolumn{9}{|c|}{ Fast food consumption } \\
\hline Model 1 & 1.43 & $1.26,1.60$ & 1.51 & $1.36,1.66$ & $1.73^{*}$ & $1.54,1.91$ & $1 \cdot 70^{*}$ & $1.54,1.86$ \\
\hline Model 2 & 1.46 & $1.28,1.63$ & 1.52 & $1.37,1.67$ & $1.73^{*}$ & $1.54,1.91$ & 1.69 & $1.53,1.85$ \\
\hline \multirow[t]{4}{*}{ Model 3} & 1.43 & $1 \cdot 26,1 \cdot 61$ & 1.51 & $1.36,1.66$ & $1 \cdot 74^{*}$ & $1.55,1.92$ & $1 \cdot 70^{*}$ & $1.54,1.86$ \\
\hline & \multicolumn{6}{|c|}{ Sleep quality $\|$} & & \\
\hline & \multicolumn{2}{|c|}{$\begin{array}{c}\text { Not at all } \\
(N \text { 604) (referent) }\end{array}$} & \multicolumn{2}{|c|}{$\begin{array}{l}\text { Somewhat } \\
(N \text { 811) }\end{array}$} & \multicolumn{2}{|c|}{$\begin{array}{l}\text { Very much } \\
(N 358)\end{array}$} & & \\
\hline & Mean & $95 \% \mathrm{Cl}$ & Mean & $95 \% \mathrm{Cl}$ & Mean & $95 \% \mathrm{Cl}$ & & \\
\hline \multicolumn{9}{|c|}{ Caffeinated beverages } \\
\hline Model 1 & 0.63 & $0.55,0.71$ & 0.72 & $0.65,0.80$ & $0.83^{\star \star}$ & $0.71,0.95$ & & \\
\hline Model 2 & 0.70 & $0.61,0.78$ & 0.72 & $0.64,0.80$ & 0.73 & $0.61,0.85$ & & \\
\hline Model 3 & 0.70 & $0.62,0.79$ & 0.72 & $0.64,0.80$ & 0.71 & $0.59,0.83$ & & \\
\hline \multicolumn{9}{|c|}{ Sugar-sweetened beverages } \\
\hline Model 1 & 0.82 & $0.73,0.92$ & 0.85 & $0.75,0.95$ & $1.06^{*}$ & $0.89,1.22$ & & \\
\hline Model 2 & 0.89 & $0.78,1.00$ & 0.85 & $0.75,0.95$ & 0.95 & $0.78,1.12$ & & \\
\hline Model 3 & 0.89 & $0.78,1.00$ & 0.85 & $0.75,0.95$ & 0.94 & $0.77,1 \cdot 10$ & & \\
\hline \multicolumn{9}{|c|}{ Sugar-free beverages } \\
\hline Model 1 & 0.30 & $0.24,0.35$ & 0.37 & $0.31,0.43$ & $0.42^{*}$ & $0.33,0.52$ & & \\
\hline Model 2 & 0.33 & $0.27,0.39$ & 0.36 & $0.30,0.42$ & 0.40 & $0.30,0.49$ & & \\
\hline Model 3 & 0.33 & $0.27,0.39$ & 0.36 & $0.31,0.42$ & 0.39 & $0.29,0.48$ & & \\
\hline \multicolumn{9}{|c|}{ Breakfast consumption } \\
\hline Model 1 & $4 \cdot 15$ & $3.93,4.37$ & 3.98 & $3 \cdot 80,4 \cdot 16$ & $3 \cdot 27^{\star \star}$ & $3.02,3.51$ & & \\
\hline Model 2 & 4.04 & $3.81,4.27$ & 3.98 & $3 \cdot 80,4 \cdot 16$ & $3.42^{\star \star}$ & $3 \cdot 16,3 \cdot 68$ & & \\
\hline Model 3 & 4.03 & $3 \cdot 81,4 \cdot 26$ & 3.98 & $3 \cdot 80,4 \cdot 16$ & $3.43^{\star \star}$ & $3 \cdot 17,3.69$ & & \\
\hline \multicolumn{9}{|c|}{ Fast food consumption } \\
\hline Model 1 & 1.50 & $1.36,1.63$ & 1.64 & $1.52,1.76$ & 1.70 & $1.49,1.92$ & & \\
\hline Model 2 & 1.59 & $1.44,1.74$ & 1.62 & $1.50,1.74$ & 1.60 & $1.37,1.82$ & & \\
\hline Model 3 & 1.59 & $1.44,1.74$ & 1.62 & $1.50,1.74$ & 1.60 & $1.37,1.82$ & & \\
\hline
\end{tabular}

Model 1 adjusted for age, sex, race/ethnicity, education and marital status.

Model 2 added depression and physical activity.

Model 3 added time in bed.

Beverages measured in servings per day; breakfast and fast food servings per week.

${ }^{\star} P<0.05,{ }^{\star \star} P<0.01,{ }^{\star \star *} P<0.001$ compared with the referent category.

†Caffeinated beverages were defined as low-calorie beverages with caffeine (e.g. Diet Coke, Diet Mountain Dew), carbonated beverages with caffeine and sugar (e.g. Coke, Pepsi, Mountain Dew, Dr. Pepper), tea with caffeine including green tea, coffee with caffeine and dairy coffee drink (e.g. cappuccino).

$\ddagger$ Sugar-sweetened beverages were defined as carbonated beverages with caffeine and sugar, other carbonated beverages with sugar (e.g. 7-Up, Root Beer, Ginger Ale, Caffeine-Free Coke) and other sugared beverages (punch, lemonade, sports drinks or sugared ice tea).

$\S$ Sugar-free beverages were defined as low-calorie beverages with caffeine and other low-calorie beverages without caffeine (e.g. Diet 7-Up).

$\|$ Sleep quality was assessed via the following question: 'During the past 12 months, how often have you been bothered or troubled by having trouble going to sleep or staying asleep?'

asleep were more likely to consume sports drinks than those who reported no difficulty ( $\mathrm{PR}=1.24$ (95\% CI 0.99, 1.49)).

Sensitivity analyses were also performed for all outcomes with an additional time in bed category: $<6 \mathrm{~h}$ (see online supplementary material, Supplemental Tables 1 and 2). Although precision was poor, those who slept for $<6 \mathrm{~h}$ per night drank approximately 0.35 more daily servings of caffeinated drinks, 0.45 more daily servings of sugar-sweetened beverages and skipped breakfast one additional time every $10 \mathrm{~d}$ compared with those who slept $7-<8 \mathrm{~h}$. Those who slept $<6 \mathrm{~h}$ were also more likely to consume energy drinks $(P R=1.55(95 \%$ CI $0.59,2.50))$. No statistically significant associations were found for sugar-free beverages, fast food or sports drinks. In the second sensitivity analysis which adjusted for BMI in an additional model, no appreciable changes in the estimates were detected (online supplementary material, Supplemental Tables 3 and 4).

\section{Discussion}

In this population-based study of young adults, we provide new evidence that sleep characteristics beyond time in bed are associated with selected eating and drinking behaviours. Late sleep timing was most consistently associated with poor 
Table 3 Mean dietary intakes (95\% Cl) by sleep indices stratified by sex: young adults aged 20-30 years, Minneapolis/St. Paul metropolitan area of Minnesota, USA, 2008-2009, Project EAT (Eating and Activity in Teens and Young Adults)

\begin{tabular}{|c|c|c|c|c|c|c|c|c|c|}
\hline & \multicolumn{8}{|c|}{ Time in bed } & \multirow[b]{3}{*}{$P$ for interaction } \\
\hline & \multicolumn{2}{|c|}{$<7 \mathrm{~h}$ per night } & \multicolumn{2}{|c|}{$\begin{array}{c}7-<8 \mathrm{~h} \text { per night } \\
\text { (referent) }\end{array}$} & \multicolumn{2}{|c|}{$8-<9 \mathrm{~h}$ per night } & \multicolumn{2}{|c|}{$\geq 9 \mathrm{~h}$ per night } & \\
\hline & Mean & $95 \% \mathrm{Cl}$ & Mean & $95 \% \mathrm{Cl}$ & Mean & $95 \% \mathrm{Cl}$ & Mean & $95 \% \mathrm{Cl}$ & \\
\hline \multicolumn{10}{|c|}{ Breakfast consumption } \\
\hline Model 1 & & & & & & & & & 0.01 \\
\hline Men & 3.90 & $3.40,4.39$ & 3.44 & $3 \cdot 14,3 \cdot 74$ & 3.83 & $3.51,4.15$ & 3.15 & $2 \cdot 76,3.53$ & \\
\hline Women & $3.49^{\star \star}$ & $3.00,3.98$ & 4.36 & $4.05,4.66$ & 4.23 & $3.98,4.49$ & 3.99 & $3.65,4.32$ & \\
\hline \multirow{6}{*}{$\begin{array}{l}\text { Model } 2 \\
\text { Men } \\
\text { Women }\end{array}$} & & & & & & & & & 0.01 \\
\hline & 3.78 & $3 \cdot 30,4 \cdot 25$ & 3.35 & $3.05,3.65$ & 3.71 & $3.37,4.04$ & 3.08 & $2 \cdot 70,3.46$ & \\
\hline & $3 \cdot 60^{\star *}$ & $3 \cdot 10,4 \cdot 10$ & 4.41 & $4 \cdot 11,4 \cdot 71$ & $4 \cdot 28$ & $4.03,4.53$ & $4 \cdot 12$ & $3 \cdot 78,4.46$ & \\
\hline & \multicolumn{8}{|c|}{ Sleep variability } & \\
\hline & \multicolumn{2}{|c|}{$<0.5 \mathrm{~h}$ (referent) } & \multicolumn{2}{|c|}{$0.5-<1 \mathrm{~h}$} & \multicolumn{2}{|c|}{$1-1.5 \mathrm{~h}$} & \multicolumn{2}{|c|}{$>1.5 \mathrm{~h}$} & \\
\hline & Mean & $95 \% \mathrm{Cl}$ & Mean & $95 \% \mathrm{Cl}$ & Mean & $95 \% \mathrm{Cl}$ & Mean & $95 \% \mathrm{Cl}$ & $P$ for interaction \\
\hline \multirow{2}{*}{\multicolumn{10}{|c|}{ Breakfast consumption }} \\
\hline & & & & & & & & & $<0.01$ \\
\hline Men & 3.84 & $3.44,4.25$ & 3.42 & $3.06,3.77$ & 3.75 & $3.43,4.08$ & 3.38 & $3.02,3.74$ & \\
\hline Women & 3.77 & $3.37,4.16$ & $4 \cdot 62^{\star \star}$ & $4.29,4.96$ & $4 \cdot 10$ & $3.81,4.39$ & 4.02 & $3 \cdot 73,4 \cdot 31$ & \\
\hline \multirow{9}{*}{$\begin{array}{l}\text { Model } 2 \\
\text { Men } \\
\text { Women } \\
\text { Model } 3 \\
\text { Men } \\
\text { Women }\end{array}$} & & & & & & & & & $<0.01$ \\
\hline & $3 \cdot 71$ & $3 \cdot 30,4 \cdot 12$ & $3 \cdot 32$ & $2.97,3.68$ & 3.66 & $3.34,3.99$ & 3.28 & $2.93,3.64$ & \\
\hline & $3 \cdot 83$ & $3 \cdot 44,4 \cdot 21$ & $4.73^{\star \star \star}$ & $4.41,5.06$ & $4 \cdot 14$ & $3.85,4.43$ & $4 \cdot 15$ & $3.86,4.44$ & \\
\hline & & & & & & & & & $<0.01$ \\
\hline & $3 \cdot 75$ & $3 \cdot 35,4 \cdot 16$ & $3 \cdot 25$ & $2 \cdot 89,3.60$ & 3.63 & $3.31,3.95$ & $3 \cdot 27$ & $2 \cdot 92,3.61$ & \\
\hline & 3.83 & $3 \cdot 44,4 \cdot 22$ & $4 \cdot 73^{\star \star \star}$ & $4.41,5.04$ & $4 \cdot 12$ & $3.83,4.41$ & $4 \cdot 14$ & $3 \cdot 84,4.44$ & \\
\hline & \multicolumn{8}{|c|}{ Sleep timing } & \\
\hline & \multicolumn{2}{|c|}{$\leq 22.30$ hours (referent) } & \multicolumn{2}{|c|}{ 22.30-23.30 hours } & \multicolumn{2}{|c|}{$23.30-00.30$ hours } & \multicolumn{2}{|c|}{$>00.30$ hours } & \\
\hline & Mean & $95 \% \mathrm{Cl}$ & Mean & $95 \% \mathrm{Cl}$ & Mean & $95 \% \mathrm{Cl}$ & Mean & $95 \% \mathrm{Cl}$ & $P$ for interaction \\
\hline \multicolumn{10}{|c|}{ Caffeinated beverages $\dagger$} \\
\hline Model 1 & & & & & & & & & $<0.01$ \\
\hline Men & 0.43 & $0.30,0.55$ & $0.70^{\star \star \star}$ & $0.58,0.82$ & $0 \cdot 80^{\star \star \star}$ & $0.63,0.97$ & $0.93^{\star \star \star}$ & $0.78,1.07$ & \\
\hline Women & $0 \cdot 70$ & $0.57,0.84$ & 0.64 & $0.51,0.77$ & 0.67 & $0.53,0.81$ & 0.72 & $0.60,0.84$ & \\
\hline Model 2 & & & & & & & & & $<0.01$ \\
\hline Men & 0.56 & $0.41,0.71$ & $0.76^{\star}$ & $0.63,0.89$ & $0 \cdot 89^{\star \star}$ & $0.72,1.07$ & $0.97^{\star \star \star}$ & $0.82,1.11$ & \\
\hline Women & 0.67 & $0.54,0.81$ & 0.63 & $0.50,0.75$ & 0.61 & $0.48,0.75$ & 0.67 & $0.55,0.79$ & \\
\hline
\end{tabular}


Table 3 Continued

\begin{tabular}{|c|c|c|c|c|c|c|c|c|c|}
\hline & \multicolumn{8}{|c|}{ Sleep timing } & \multirow[b]{3}{*}{$P$ for interaction } \\
\hline & \multicolumn{2}{|c|}{$\leq 22.30$ hours (referent) } & \multicolumn{2}{|c|}{ 22.30-23.30 hours } & \multicolumn{2}{|c|}{$23.30-00.30$ hours } & \multicolumn{2}{|c|}{$>00.30$ hours } & \\
\hline & Mean & $95 \% \mathrm{Cl}$ & Mean & $95 \% \mathrm{Cl}$ & Mean & $95 \% \mathrm{Cl}$ & Mean & $95 \% \mathrm{Cl}$ & \\
\hline Model 3 & & & & & & & & & $<0.01$ \\
\hline Men & 0.60 & $0.44,0.75$ & 0.78 & $0.65,0.91$ & $0.89^{\star \star}$ & $0.71,1.06$ & $0.94^{\star \star}$ & $0.80,1.09$ & \\
\hline Women & 0.68 & $0.54,0.81$ & 0.63 & $0.50,0.76$ & 0.62 & $0.48,0.76$ & 0.66 & $0.54,0.78$ & \\
\hline \multicolumn{10}{|c|}{ Sugar-free beverages $\ddagger$} \\
\hline Model 1 & & & & & & & & & $<0.01$ \\
\hline Men & 0.14 & $0.06,0.22$ & $0 \cdot 32^{\star \star}$ & $0.24,0.41$ & $0.36^{\star \star}$ & $0.23,0.48$ & $0.39^{\star \star}$ & $0.28,0.49$ & \\
\hline & 0.47 & $0.36,0.57$ & 0.35 & $0.26,0.44$ & 0.42 & $0.29,0.55$ & 0.35 & $0.26,0.44$ & \\
\hline \multicolumn{9}{|l|}{ Model 2} & $<0.01$ \\
\hline Men & 0.16 & $0.08,0.25$ & $0.34^{\star \star}$ & $0.25,0.44$ & $0.38^{\star \star}$ & $0.24,0.51$ & $0.40^{\star \star \star}$ & $0.29,0.50$ & \\
\hline Women & 0.47 & $0.37,0.58$ & 0.35 & $0.26,0.44$ & 0.41 & $0.28,0.53$ & 0.34 & $0.25,0.43$ & \\
\hline \multicolumn{9}{|l|}{ Model 3} & $<0.01$ \\
\hline Men & 0.20 & $0.10,0.29$ & $0.35^{\star}$ & $0.25,0.45$ & $0.36^{\star}$ & $0.23,0.49$ & $0.37^{\star}$ & $0.27,0.47$ & \\
\hline Women & 0.48 & $0.37,0.58$ & 0.35 & $0.26,0.44$ & 0.41 & $0.28,0.53$ & 0.34 & $0.24,0.43$ & \\
\hline
\end{tabular}

Model 1 adjusted for age, race/ethnicity, education and marital status.

Model 2 added depression, and physical activity.

Model 3 added time in bed.

Breakfast consumption measured in servings per week; beverages in servings per day.

${ }^{*} P<0.05,{ }^{* \star} P<0.01,{ }^{* \star *} P<0.001$ compared with the referent category.

†Caffeinated beverages were defined as low-calorie beverages with caffeine (e.g. Diet Coke, Diet Mountain Dew), carbonated beverages with caffeine and sugar (e.g. Coke, Pepsi, Mountain Dew, Dr. Pepper), tea with caffeine including green tea, coffee with caffeine and dairy coffee drink (e.g. cappuccino).

¥Sugar-free beverages were defined as low-calorie beverages with caffeine and other low-calorie beverages without caffeine (e.g. Diet 7-Up). 
Table 4 Prevalence ratios $(95 \% \mathrm{Cl})$ for intakes of energy drinks and sports drinks by sleep duration, variability, timing and quality: young adults aged 20-30 years, Minneapolis/St. Paul metropolitan area of Minnesota, USA, 2008-2009, Project EAT (Eating and Activity in Teens and Young Adults)

\begin{tabular}{|c|c|c|c|c|c|c|c|c|}
\hline & \multicolumn{8}{|c|}{ Time in bed } \\
\hline & \multicolumn{2}{|c|}{$<7 \mathrm{~h}$ per night $(N 203)$} & \multicolumn{2}{|c|}{$7-<8 \mathrm{~h}$ per night $(N 471)$} & \multicolumn{2}{|c|}{$8-<9 \mathrm{~h}$ per night $(N 642)$} & \multicolumn{2}{|c|}{$\geq 9 \mathrm{~h}$ per night $(N 457$} \\
\hline & PR & $95 \% \mathrm{Cl}$ & PR & $95 \% \mathrm{Cl}$ & PR & $95 \% \mathrm{Cl}$ & PR & $95 \% \mathrm{Cl}$ \\
\hline \multicolumn{9}{|c|}{ Energy drinks $†$} \\
\hline Model 1 & 1.45 & $0.95,1.94$ & 1.00 & Ref. & 1.09 & $0.79,1.39$ & 1.03 & $0.71,1.35$ \\
\hline Model 2 & 1.39 & $0.91,1.87$ & 1.00 & Ref. & 1.08 & $0.79,1.38$ & 1.01 & $0.71,1.32$ \\
\hline \multicolumn{9}{|c|}{ Sports drinks $\dagger$} \\
\hline Model 1 & 0.97 & $0.74,1 \cdot 20$ & 1.00 & Ref. & 0.86 & $0.71,1.02$ & $0.80^{*}$ & $0.62,0.97$ \\
\hline \multirow[t]{4}{*}{ Model 2} & 0.94 & $0.71,1 \cdot 18$ & 1.00 & Ref. & $0.85^{\star}$ & $0.70,0.99$ & $0 \cdot 80^{*}$ & $0.63,0.98$ \\
\hline & \multicolumn{8}{|c|}{ Sleep variability } \\
\hline & \multicolumn{2}{|c|}{$<0.5 \mathrm{~h}(N 339)$} & \multicolumn{2}{|c|}{$0.5-<1 \mathrm{~h}(N 364)$} & \multicolumn{2}{|c|}{$1-1.5 \mathrm{~h}(N 571)$} & \multicolumn{2}{|c|}{$>1.5 \mathrm{~h}(N 500)$} \\
\hline & PR & $95 \% \mathrm{Cl}$ & PR & $95 \% \mathrm{Cl}$ & PR & $95 \% \mathrm{Cl}$ & PR & $95 \% \mathrm{Cl}$ \\
\hline \multicolumn{9}{|c|}{ Energy drinks } \\
\hline Model 1 & 1.00 & Ref. & $1.59^{* *}$ & $1 \cdot 04,2 \cdot 14$ & 1.30 & $0.88,1.73$ & $1.40^{*}$ & $0.93,1.87$ \\
\hline Model 2 & 1.00 & Ref. & $1.54^{*}$ & $1.02,2.07$ & 1.29 & $0.87,1.70$ & 1.36 & $0.90,1.81$ \\
\hline Model 3 & 1.00 & Ref. & $1.53^{*}$ & $1.01,2.04$ & 1.29 & $0.87,1.71$ & 1.35 & $0.89,1.81$ \\
\hline \multicolumn{9}{|c|}{ Sports drinks } \\
\hline Model 1 & 1.00 & Ref. & 1.08 & $0.84,1.33$ & 1.01 & $0.80,1.23$ & 0.93 & $0.71,1.14$ \\
\hline Model 2 & 1.00 & Ref. & 1.06 & $0.82,1.30$ & 0.99 & $0.79,1.20$ & 0.92 & $0.71,1.13$ \\
\hline Model 3 & 1.00 & Ref. & 1.02 & $0.79,1.24$ & 0.95 & $0.76,1 \cdot 15$ & 0.87 & $0.68,1.07$ \\
\hline
\end{tabular}

Sleep timing

\begin{tabular}{|c|c|c|c|c|c|c|c|c|}
\hline & \multicolumn{2}{|c|}{$\leq 22.30$ hours $(N 237)$} & \multicolumn{2}{|c|}{$22.30-23.30$ hours $(N 497)$} & \multicolumn{2}{|c|}{$23.30-00.30$ hours $(N 390)$} & \multicolumn{2}{|c|}{$>00.30$ hours $(N 560)$} \\
\hline & PR & $95 \% \mathrm{Cl}$ & PR & $95 \% \mathrm{Cl}$ & PR & $95 \% \mathrm{Cl}$ & PR & $95 \% \mathrm{Cl}$ \\
\hline \multicolumn{9}{|c|}{ Energy drinks } \\
\hline Model 1 & 1.00 & Ref. & 1.44 & $0.86,2.01$ & $1.63^{*}$ & $0.97,2.29$ & $1 \cdot 83^{\star *}$ & $1 \cdot 10,2.55$ \\
\hline Model 2 & 1.00 & Ref. & 1.36 & $0.82,1.90$ & $1.53^{*}$ & $0.92,2 \cdot 15$ & $1.71^{\star *}$ & $1.04,2.38$ \\
\hline Model 3 & 1.00 & Ref. & 1.35 & $0.82,1.89$ & $1.50^{\star}$ & $0.90,2.11$ & $1 \cdot 65^{\star *}$ & $0.99,2 \cdot 30$ \\
\hline \multicolumn{9}{|c|}{ Sports drinks } \\
\hline Model 1 & 1.00 & Ref. & 1.07 & $0.80,1.34$ & 1.07 & $0.79,1.35$ & 1.22 & $0.92,1.51$ \\
\hline Model 2 & 1.00 & Ref. & 1.03 & $0.78,1.28$ & 1.04 & $0.77,1.30$ & $1 \cdot 18$ & $0.90,1.46$ \\
\hline Model 3 & 1.00 & Ref. & 1.01 & $0.76,1.26$ & 1.01 & $0.75,1.26$ & $1 \cdot 15$ & $0.87,1.43$ \\
\hline
\end{tabular}

Sleep quality

\begin{tabular}{|c|c|c|c|c|c|c|}
\hline & \multicolumn{2}{|c|}{ Not at all (N 604) } & \multicolumn{2}{|c|}{ Somewhat (N 811) } & \multicolumn{2}{|c|}{ Very much (N 358) } \\
\hline & PR & $95 \% \mathrm{Cl}$ & PR & $95 \% \mathrm{Cl}$ & PR & $95 \% \mathrm{Cl}$ \\
\hline \multicolumn{7}{|c|}{ Energy drinks } \\
\hline Model 1 & 1.00 & Ref. & $1.42^{* \star}$ & $1.06,1.78$ & $1.96^{\star \star \star}$ & $1 \cdot 40,2 \cdot 51$ \\
\hline Model 2 & 1.00 & Ref. & $1.38^{*}$ & $1.01,1.74$ & $1.84^{\star \star \star}$ & $1 \cdot 28,2.40$ \\
\hline Model 3 & 1.00 & Ref. & $1 \cdot 37^{*}$ & $1.01,1.74$ & $1 \cdot 79^{\star \star \star}$ & $1 \cdot 24,2 \cdot 34$ \\
\hline \multicolumn{7}{|c|}{ Sports drinks } \\
\hline Model 1 & 1.00 & Ref. & 1.00 & $0.83,1.17$ & $1 \cdot 24^{*}$ & $0.99,1.49$ \\
\hline Model 2 & 1.00 & Ref. & 0.99 & $0.82,1.17$ & $1 \cdot 27^{\star}$ & $1.00,1.55$ \\
\hline Model 3 & 1.00 & Ref. & 1.00 & $0.83,1.18$ & $1 \cdot 28^{*}$ & $1.00,1.56$ \\
\hline
\end{tabular}

PR, prevalence ratio; Ref., referent category.

Model 1 adjusted for age, sex, race/ethnicity, education and marital status.

Model 2 added depression and physical activity.

Model 3 added sleep duration.

${ }^{\star} P<0.05,{ }^{\star *} P<0.01,{ }^{\star \star \star} P<0.001$.

†Energy and sports drink consumption defined as at least one drink per week $v$. less than one drink per week.

¥Sleep quality was assessed via the following question: 'During the past 12 months, how often have you been bothered or troubled by having trouble going to sleep or staying asleep?'

eating and drinking behaviours, including consumption of energy drinks, sugar-sweetened beverages, fast food and breakfast skipping. Fewer associations were found for other sleep indices.
In the present study, going to sleep late was directly associated with four of the seven poor eating and drinking behaviours, including more frequent consumption of energy drinks and sugar-sweetened beverages, and frequency of 
eating at fast-food restaurants, as well as breakfast skipping. These findings are consistent with another small crosssectional study, where actigraphy-measured late sleep timing was associated with more servings of full-calorie soda and fast food per week, although results were not adjusted for confounders ${ }^{(18)}$. Previous research also found that delaying bedtime was not associated with more caffeine use $^{(41)}$, while we found a small association between sleep timing and caffeinated beverages that was no longer significant after adjustments for depressive symptoms, alcohol and physical activity. Research on other dietary measures has found actigraphy-measured sleep timing was not associated with the Alternative Healthy Eating Index-2010 or any of its components ${ }^{(42)}$. An alternative explanation for these findings may be night eating syndrome, which is characterized by 'recurrent episodes of night eating, as manifested by eating after awakening from sleep or by excessive food consumption after the evening meal ${ }^{\text {(43) }}$. However, this syndrome requires that sleep-wake changes not be the best explanation for the night eating.

Less time in bed, an approximation of sleep duration, was associated with more caffeinated beverage consumption, while more time in bed was associated with less sports drink consumption. The relationship between sleep duration (or time in bed) and eating and drinking behaviours has been examined more frequently in previous literature than the relationships between other sleep indices and eating and drinking behaviours. Observational studies on time in bed or sleep duration and caffeinated beverages have similarly found inverse associations $^{(15,41,44,45)}$, although one found no association ${ }^{(45)}$. Additionally, those who slept fewer than $6 \mathrm{~h}$ per night were more likely to skip breakfast ${ }^{(15)}$ and consume sugarsweetened beverages, including caffeinated beverages, than those who slept $7-8 \mathrm{~h}$ per night ${ }^{(46)}$. In the current study, the proportion sleeping at least $7 \mathrm{~h}$ per night was $88.5 \%$, which contrasts with national survey data where only $67 \cdot 8 \%$ of $18-24$-year-olds and $62 \cdot 1 \%$ of $25-34$-yearolds reported sleeping at least $7 \mathrm{~h}$ per night, although the self-report methods of these two studies were different ${ }^{(9)}$. Because of the narrow distribution of sleep in our sample, we did not have sufficient precision to consider more extreme categories of shorter sleep duration as the primary exposure, although in sensitivity analyses we showed that those who slept fewer than $6 \mathrm{~h}$ per night skipped more breakfast and consumed more sugar-sweetened beverages than those sleeping 7-8 h per night. Studies of adults and adolescents have reported that those with short sleep duration ate fast food more often than those meeting sleep recommendations ${ }^{(16,47)}$, a finding not replicated in our study.

In the present study, poor sleep quality was significantly associated with six of the seven poor eating and drinking behaviours, but associations remained only for energy drinks, sports drinks and skipping breakfast after adjustment for depressive symptoms, alcohol and physical activity. Previous research on sleep quality and intake of caffeine/energy drinks has been mixed, with studies finding null ${ }^{(44,48)}$ or inverse associations ${ }^{(49-52)}$. However, a study of Japanese female workers found that poor sleep quality was associated with greater sugar-sweetened beverage consumption and breakfast skipping ${ }^{(53)}$. Studies involving other dietary and eating measures have found associations between poor sleep quality and low intake of vegetables and fish ${ }^{(53)}$, as well as lower adherence to the Mediterranean diet.

Sleep variability has been rarely measured in population-based studies, especially in relation to diet. In the present study high sleep variability was associated with greater fast-food and energy drink consumption. Previous studies have found positive associations between sleep variability and obesity ${ }^{(54,55)}$, including a mediating influence of diet variables ${ }^{(56)}$, although no association was found in a previous Project EAT analysis ${ }^{(25)}$.

Overall, in the present study, associations between sleep and eating and drinking behaviours were not consistent across indices. This may reflect measurement error, as described below, or may indicate unique dimensions of sleep. While some aspects of sleep, like duration, can be measured objectively, self-reported sleep quality is inherently subjective, and thus may capture a different aspect of the sleep process or reflect differences in self-reporting. In the present study, the four sleep indices used were weakly correlated $(r<0 \cdot 2)$. Future research should continue to focus on multiple dimensions to obtain a complete picture of sleep. Prospective data are also needed to further elucidate the relationship between sleep and dietary intake.

We observed effect modification by sex for some exposure-outcome combinations. Previous laboratory research also found differences, where men consumed more daily energy than women after sleep restriction ${ }^{(39)}$. Differences could be due to gender biology (e.g. levels of hormones), differences in social desirability that impact self-reporting, or ways that society influences coping during sleep restriction periods differentially by gender ${ }^{(57)}$. In general, sleep duration is longer in women than men $^{(58,59)}$.

The relationship between sleep and diet is likely complex, and potentially bidirectional. Caffeinated drinks, such as soda and energy drinks, block adenosine receptors, which prevent the sleep-promoting effects of adenosine and thus reduce sleep duration. However, people who are sleep deprived may consume more caffeinated drinks to feel more alert ${ }^{(60)}$. Short sleep duration may also influence diet by providing more time and opportunities for eating and drinking, allowing people to be more sensitive to food rewards, decreasing restraint and changing concentrations of hormones that influence appetite, such as leptin and ghrelin ${ }^{(61)}$. However, limited evidence also suggests that nutrients that help synthesize serotonin may also promote sleep ${ }^{(62)}$. 
Other sleep indices likely act through similar pathways. Although the mechanisms for associations between sleep timing and diet are not fully elucidated, possible mechanisms include circadian disruption and greater exposure to light at night ${ }^{(63,64)}$. People with high sleep variability may also have irregular eating patterns due to variation in their sleep-wake pattern, which may contribute to irregularity in the synchronization of eating and sleep timing ${ }^{(54)}$.

The present study has several limitations, including measurement error in the sleep and dietary variables, which were both assessed via survey. Self-reported and objectively measured sleep are moderately correlated ${ }^{(65-67)}$, but the degree of correlation varies by important confounders such as obesity and depression. Dietary intake was self-reported and likely represents an underestimation of intake. Previous research has found moderate to high reproducibility and validity of FFQ compared with diet records, although both measures are self-reported ${ }^{(34)}$. Combined, these errors in measurement may have biased the estimates towards (likely) or away from the null (less likely). Additionally, the present crosssectional study inherently offers no information on temporality and the causal pathway between sleep and diet is at times unclear, particularly for caffeine and energy drinks. These associations between sleep and diet may also be due to a shared cause. We also performed many statistical tests, so it is possible that some of the results may be due to chance.

Despite these limitations, our study has several strengths. Quality observational studies on sleep duration and dietary intake are limited, due to an emphasis on short-term experiments conducted in sleep labs, which do not provide information on habitual behaviours among free-living adults. Another strength of the present study is the diverse and population-based sample of young adults. Use of this sample aligns with the 2016 American Heart Association Scientific Statement that highlighted the need for sleep studies to include diverse populations ${ }^{(24)}$. Additionally, the measurement instruments used in the study employed multiple indices of sleep and diet, which allowed the capture of these behaviours in several different dimensions.

Sleep and diet are both inherently vital health behaviours. Short sleep duration is highly prevalent, especially among young adults ${ }^{(9)}$. Dietary quality may be improving ${ }^{(68)}$, but the vast majority of the US population is still not meeting dietary recommendations. Although the present study found some cross-sectional associations between sleep and eating behaviours, particularly as related to sleep timing, many associations were not significant, and further longitudinal and randomized studies with objective measures of sleep are needed to clarify the directionality of the sleep-diet relationship. Young adults often experience significant changes in their establishment of an independent life, including attainment of higher education, new employment, getting married and having children. As such, if the relationships found between sleep and eating behaviours herein are causal, sleep-friendly interventions and policies may have the potential, along with other risk factors, to reduce obesity in this population. Identifying effective obesity prevention measures for young adults is particularly important, as at this age range there is the potential to set long-term health habits. Additionally, nutrition professionals could informally screen for poor sleep or a sleep disorder and make referrals to a sleep specialist as necessary ${ }^{(69)}$.

\section{Acknowledgements}

Financial support: This research was supported by the National Heart, Lung, and Blood Institute (NHLBI) (grant numbers R01-HL-084064, T32-HL-007779 and T32-HL082610). The NHLBI had no role in the design, analysis or writing of this article. Conflict of interest: The authors report no conflicts of interest. Authorship: R.P.O. formulated the research question, analysed the data and wrote the manuscript. P.L.L. helped formulated the research, provided input on the analysis, and read and reviewed the manuscript. R.W. and M.N.L. helped formulate the research question and read and reviewed the manuscript. N.L. read and reviewed the manuscript. D.N.-S. designed and obtained grant funding for the study and read and reviewed the manuscript. Ethics of buman subject participation: The University of Minnesota IRB approved all study protocols and participants provided informed consent.

\section{Supplementary material}

To view supplementary material for this article, please visit https://doi.org/10.1017/S1368980017003536

\section{References}

1. Consensus Conference Panel, Watson NF, Badr MS et al. (2015) Joint consensus statement of the American Academy of Sleep Medicine and Sleep Research Society on the recommended amount of sleep for a healthy adult: methodology and discussion. Sleep 38, 1161-1183.

2. Hirshkowitz M, Whiton K, Albert SM et al. (2015) National Sleep Foundation's sleep time duration recommendations: methodology and results summary. Sleep Health 1, 40-43.

3. Centers for Disease Control and Prevention (2017) Sleep and Sleep Disorders. http://www.cdc.gov/features/sleep/ (accessed October 2017).

4. Cappuccio FP, Cooper D, D'Elia L et al. (2011) Sleep duration predicts cardiovascular outcomes: a systematic review and meta-analysis of prospective studies. Eur Heart $J$ 32, 1484-1492.

5. Zhai L, Zhang H \& Zhang D (2015) Sleep duration and depression among adults: a meta-analysis of prospective studies. Depress Anxiety 32, 664-670.

6. Bayan-Bravo A, Perez-Tasigchana RF, Sayon-Orea C et al. (2017) Combined impact of traditional and non-traditional 
healthy behaviors on health-related quality of life: a prospective study in older adults. PLoS One 12, e0170513.

7. Magee CA, Caputi P \& Iverson DC (2011) Relationships between self-rated health, quality of life and sleep duration in middle aged and elderly Australians. Sleep Med 12, 346-350.

8. Chen X, Gelaye B \& Williams MA (2014) Sleep characteristics and health-related quality of life among a national sample of American young adults: assessment of possible health disparities. Qual Life Res 23, 613-625.

9. Liu Y, Wheaton AG, Chapman DP et al. (2016) Prevalence of healthy sleep duration among adults - United States, 2014. MMWR Morb Mortal Wkly Rep 65, 137-141.

10. Weiss A, Xu F, Storfer-Isser A et al. (2010) The association of sleep duration with adolescents' fat and carbohydrate consumption. Sleep 33, 1201-1209.

11. Bel S, Michels N, De Vriendt T et al. (2013) Association between self-reported sleep duration and dietary quality in European adolescents. Br J Nutr 110, 949-959.

12. Capers PL, Fobian AD, Kaiser KA et al. (2015) A systemic review and meta-analysis of randomized controlled trials of the impact of sleep duration on adiposity and components of energy balance. Obes Rev 16, 771-782.

13. Patel SR \& Hu FB (2008) Short sleep duration and weight gain: a systematic review. Obesity (Silver Spring) 16, 643-653.

14. Magee L \& Hale L (2012) Longitudinal associations between sleep duration and subsequent weight gain: a systematic review. Sleep Med Rev 16, 231-241.

15. Kant AK \& Graubard BI (2014) Association of self-reported sleep duration with eating behaviors of American adults: NHANES 2005-2010. Am J Clin Nutr 100, 938-947.

16. Stamatakis KA \& Brownson RC (2008) Sleep duration and obesity-related risk factors in the rural Midwest. Prev Med 46, 439-444.

17. Quick V, Byrd-Bredbenner C, Shoff S et al. (2016) Relationships of sleep duration with weight-related behaviors of US college students. Behav Sleep Med 14, 565-580.

18. Baron KG, Reid KJ, Kern AS et al. (2011) Role of sleep timing in caloric intake and BMI. Obesity (Silver Spring) 19 1374-1381.

19. Hale L \& Do DP (2007) Racial differences in self-reports of sleep duration in a population-based study. Sleep 30, 1096-1103.

20. Whinnery J, Jackson N, Rattanaumpawan P et al. (2014) Short and long sleep duration associated with race/ethnicity, sociodemographics, and socioeconomic position. Sleep 37, 601-611.

21. Stamatakis KA, Kaplan GA \& Roberts RE (2007) Short sleep duration across income, education, and race/ethnic groups: population prevalence and growing disparities during 34 years of follow-up. Ann Epidemiol 17, 948-955.

22. Grandner MA, Petrov ME, Rattanaumpawan P et al. (2013) Sleep symptoms, race/ethnicity, and socioeconomic position. J Clin Sleep Med 9, 897-905; 905A-905D.

23. Grandner MA, Patel NP, Gehrman PR et al. (2010) Who gets the best sleep? Ethnic and socioeconomic factors related to sleep complaints. Sleep Med 11, 470-478.

24. St-Onge MP, Grandner MA, Brown D et al. (2016) Sleep duration and quality: impact on lifestyle behaviors and cardiometabolic health: a scientific statement from the American Heart Association. Circulation 134, e367-e386.

25. Meyer KA, Wall MM, Larson NI et al. (2012) Sleep duration and BMI in a sample of young adults. Obesity (Silver Spring) 20, 1279-1287.

26. Neumark-Sztainer D, Story M, Hannan PJ et al. (2002) Overweight status and eating patterns among adolescents: where do youths stand in comparison with the healthy people 2010 objectives? Am J Public Health 92, 844-851.

27. Larson N, Neumark-Sztainer D, Harwood EM et al. (2011) Do young adults participate in surveys that 'go green'?
Response rates to a web and mailed survey of weight-related health behaviors. Int J Child Health Hum Dev 4, 225-231.

28. Larson N, Neumark-Sztainer D, Story M et al. (2011) Identifying correlates of young adults' weight behavior: survey development. Am J Health Behav 35, 712-725.

29. Pasch KE, Laska MN, Lytle LA et al. (2010) Adolescent sleep, risk behaviors, and depressive symptoms: are they linked? Am J Health Behav 34, 237-248.

30. Lytle LA, Pasch KE \& Farbakhsh K (2011) The relationship between sleep and weight in a sample of adolescents. Obesity (Silver Spring) 19, 324-331.

31. Wolfson AR, Carskadon MA, Acebo C et al. (2003) Evidence for the validity of a sleep habits survey for adolescents. Sleep 26, 213-216.

32. Kandel DB \& Davies M (1982) Epidemiology of depressive mood in adolescents: an empirical study. Arch Gen Psychiatry 39. $1205-1212$.

33. Larson N, Laska MN, Story M et al. (2015) Sports and energy drink consumption are linked to health-risk behaviours among young adults. Public Health Nutr 18, 2794-2803.

34. Feskanich D, Rimm EB, Giovannucci EL et al. (1993) Reproducibility and validity of food intake measurements from a semiquantitative food frequency questionnaire. $J \mathrm{Am}$ Diet Assoc 93, 790-796.

35. Godin G \& Shephard RJ (1985) A simple method to assess exercise behavior in the community. Can J Appl Sport Sci 10, 141-146.

36. Quick V, Wall M, Larson N et al. (2013) Personal, behavioral and socio-environmental predictors of overweight incidence in young adults: 10-yr longitudinal findings. Int $J$ Behav Nutr Phys Act 10, 37.

37. Kline CE (2014) The bidirectional relationship between exercise and sleep: implications for exercise adherence and sleep improvement. Am J Lifestyle Med 8, 375-379.

38. Franzen PL \& Buysse DJ (2008) Sleep disturbances and depression: risk relationships for subsequent depression and therapeutic implications. Dialogues Clin Neurosci 10, 473-481.

39. Spaeth AM, Dinges DF \& Goel N (2014) Sex and race differences in caloric intake during sleep restriction in healthy adults. Am J Clin Nutr 100, 559-566.

40. Little RJA (1986) Survey nonresponse adjustments for estimates of means. Int Stat Rev 54, 139-157.

41. Regestein Q, Natarajan V, Pavlova M et al. (2010) Sleep debt and depression in female college students. Psychiatry Res 176, 34-39.

42. Mossavar-Rahmani Y, Weng J, Wang R et al. (2017) Actigraphic sleep measures and diet quality in the Hispanic Community Health Study/Study of Latinos Sueno ancillary study. J Sleep Res. Published online: 28 March 2017. doi: 10.1111/jsr.12513.

43. American Psychiatric Association (2013) Diagnostic and Statistical Manual of Mental Disorders: DSM-5. Washington, DC: American Psychiatric Association.

44. Watson EJ, Coates AM, Kohler M et al. (2016) Caffeine consumption and sleep quality in Australian adults. Nutrients 8, E479.

45. Sanchez-Ortuno M, Moore N, Taillard J et al. (2005) Sleep duration and caffeine consumption in a French middle-aged working population. Sleep Med 6, 247-251.

46. Prather AA, Leung C, Adler NE et al. (2016) Short and sweet: associations between self-reported sleep duration and sugar-sweetened beverage consumption among adults in the United States. Sleep Health 2, 272-276.

47. Kruger AK, Reither EN, Peppard PE et al. (2014) Do sleepdeprived adolescents make less-healthy food choices? $\mathrm{Br}$ J Nutr 111, 1898-1904.

48. Lund HG, Reider BD, Whiting AB et al. (2010) Sleep patterns and predictors of disturbed sleep in a large population of college students. J Adolesc Health 46, 124-132. 
49. Reid A \& Baker FC (2008) Perceived sleep quality and sleepiness in South African university students. $S$ Afr J Psychol 38, 287-303.

50. Patrick ME, Griffin J, Huntley ED et al. (2018) Energy drinks and binge drinking predict college students' sleep quantity, quality, and tiredness. Behav Sleep Med 16, 92-105.

51. Lohsoonthorn V, Khidir H, Casillas G et al. (2013) Sleep quality and sleep patterns in relation to consumption of energy drinks, caffeinated beverages, and other stimulants among Thai college students. Sleep Breath 17, 1017-1028.

52. Sanchez SE, Martinez C, Oriol RA et al. (2013) Sleep quality, sleep patterns and consumption of energy drinks and other caffeinated beverages among Peruvian college students. Health (Irvine Calif) 5, 26-35.

53. Katagiri R, Asakura K, Kobayashi S et al. (2014) Low intake of vegetables, high intake of confectionary, and unhealthy eating habits are associated with poor sleep quality among middleaged female Japanese workers. J Occup Health 56, 359-368.

54. Patel SR, Hayes AL, Blackwell T et al. (2014) The association between sleep patterns and obesity in older adults. Int $J$ Obes (Lond) 38, 1159-1164.

55. Ogilvie RP, Redline S, Bertoni AG et al. (2016) Actigraphy measured sleep indices and adiposity: the Multi-Ethnic Study of Atherosclerosis (MESA). Sleep 39, 1701-1708.

56. He F, Bixler EO, Liao J et al. (2015) Habitual sleep variability, mediated by nutrition intake, is associated with abdominal obesity in adolescents. Sleep Med 16, 1489-1494.

57. Dzaja A, Arber S, Hislop J et al. (2005) Women's sleep in health and disease. J Psychiatr Res 39, 55-76.

58. Chen X, Wang R, Zee P et al. (2015) Racial/ethnic differences in sleep disturbances: the Multi-Ethnic Study of Atherosclerosis (MESA). Sleep 38, 877-888.
59. Lauderdale DS, Knutson KL, Yan LL et al. (2006) Objectively measured sleep characteristics among early-middle-aged adults: the CARDIA study. Am J Epidemiol 164, 5-16.

60. Roehrs T \& Roth T (2008) Caffeine: sleep and daytime sleepiness. Sleep Med Rev 12, 153-162.

61. Chaput JP (2014) Sleep patterns, diet quality and energy balance. Physiol Behav 134, 86-91.

62. Peuhkuri K, Sihvola N \& Korpela R (2012) Diet promotes sleep duration and quality. Nutr Res 32, 309-319.

63. Obayashi K, Saeki K, Iwamoto J et al. (2013) Exposure to light at night, nocturnal urinary melatonin excretion, and obesity/dyslipidemia in the elderly: a cross-sectional analysis of the HEIJO-KYO study. J Clin Endocrinol Metab 98, 337-344.

64. Ramsey KM \& Bass J (2009) Obeying the clock yields benefits for metabolism. Proc Natl Acad Sci U S A 106, 4069-4070.

65. Cespedes EM, Hu FB, Redline S et al. (2016) Comparison of self-reported sleep duration with actigraphy: results from the Hispanic Community Health Study/Study of Latinos Sueno Ancillary study. Am J Epidemiol 183, 561-573.

66. Lauderdale DS, Knutson KL, Yan LL et al. (2008) Selfreported and measured sleep duration: how similar are they? Epidemiology 19, 838-845.

67. Patel SR, Blackwell T, Ancoli-Israel S et al. (2012) Sleep characteristics of self-reported long sleepers. Sleep 35, 641-648.

68. Rehm CD, Penalvo JL, Afshin A et al. (2016) Dietary intake among US adults, 1999-2012. JAMA 315, 2542-2553.

69. Golem DL, Martin-Biggers JT, Koenings MM et al. (2014) An integrative review of sleep for nutrition professionals. $A d v$ Nutr 5, 742-759. 